\title{
Size-resolved source apportionment of particulate matter in urban Beijing during haze and non-haze episodes
}

\section{S. L. Tian et al.}

Correspondence to: Y. S. Wang (wys@mail.iap.ac.cn) and Y. P. Pan (panyuepeng@mail.iap.ac.cn)

The copyright of individual parts of the supplement might differ from the CC-BY 3.0 licence. 
Table S1 Calculation methods of main components used in mass closure study

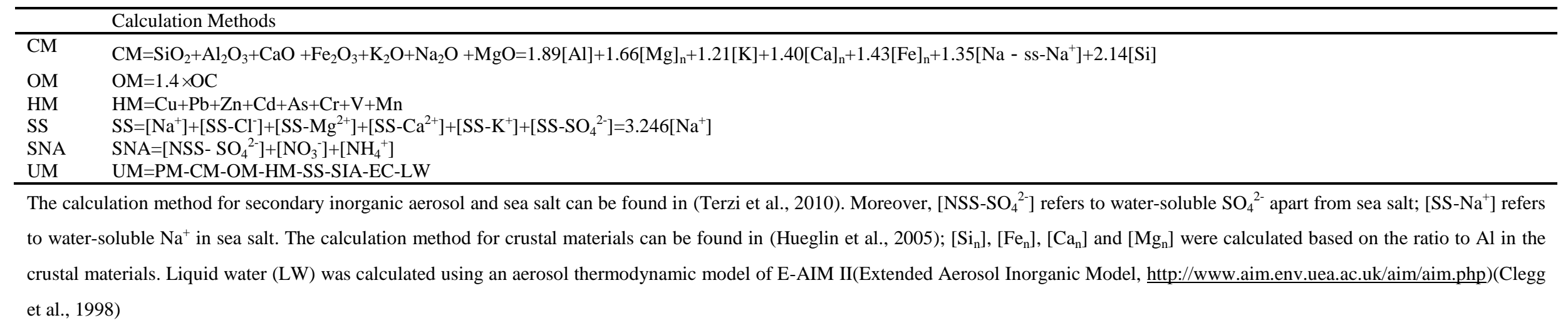

Table S2 Concentrations of different chemical compositions in size-resolved particles during entire sampling period (annual) and four seasons $\left(\mu \mathrm{g} \mathrm{m}^{-3}\right)$.

\begin{tabular}{|c|c|c|c|c|c|c|c|c|c|c|c|c|c|c|c|c|c|c|c|c|}
\hline \multirow[b]{3}{*}{ Size } & \multicolumn{4}{|c|}{ Annual } & \multicolumn{4}{|c|}{ Spring } & \multicolumn{4}{|c|}{ Summer } & \multicolumn{4}{|c|}{ Autumn } & \multicolumn{4}{|c|}{ Winter } \\
\hline & \multicolumn{2}{|c|}{ Clear } & \multicolumn{2}{|c|}{ Haze } & \multicolumn{2}{|c|}{ Clear } & \multicolumn{2}{|c|}{ Haze } & \multicolumn{2}{|c|}{ Clear } & \multicolumn{2}{|c|}{ Haze } & \multicolumn{2}{|c|}{ Clear } & \multicolumn{2}{|c|}{ Haze } & \multicolumn{2}{|c|}{ Clear } & \multicolumn{2}{|c|}{ Haze } \\
\hline & $\mathrm{PM}_{2.1}$ & $\mathrm{PM}_{2.1-9}$ & $\mathrm{PM}_{2.1}$ & $\mathrm{PM}_{2.1-9}$ & $\mathrm{PM}_{2.1}$ & $\mathrm{PM}_{2.1-9}$ & $\mathrm{PM}_{2.1}$ & $\mathrm{PM}_{2.1-9}$ & $\mathrm{PM}_{2.1}$ & $\mathrm{PM}_{2.1-9}$ & $\mathrm{PM}_{2.1}$ & $\mathrm{PM}_{2.1-9}$ & $\mathrm{PM}_{2.1}$ & $\mathrm{PM}_{2.1-9}$ & $\mathrm{PM}_{2.1}$ & $\mathrm{PM}_{2.1-9}$ & $\mathrm{PM}_{2.1}$ & $\mathrm{PM}_{2.1-9}$ & $\mathrm{PM}_{2.1}$ & $\mathrm{PM}_{2.1-9}$ \\
\hline Mass & 32.6 & 50.5 & 86.1 & 72.6 & 41.0 & 70.5 & 74.7 & 80.2 & 21.8 & 34.2 & 48.8 & 65.0 & 37.1 & 46.4 & 83.1 & 76.2 & 20.4 & 42.6 & 114.2 & 67.7 \\
\hline $\mathrm{OC}$ & 8.8 & 5.9 & 19.2 & 9.7 & 14.9 & 12.6 & 17.4 & 10.9 & 16.6 & 13.8 & 19.2 & 15.5 & 5.9 & 2.9 & 18.3 & 9.9 & 5.4 & 1.5 & 21.3 & 5.8 \\
\hline $\mathrm{EC}$ & 1.2 & 0.4 & 2.6 & 1.0 & 1.1 & 0.3 & 1.5 & 0.7 & 1.2 & 0.5 & 1.3 & 0.7 & 1.5 & 0.5 & 1.9 & 1.2 & 0.8 & 0.1 & 4.5 & 1.0 \\
\hline $\mathrm{Na}^{+}$ & 0.4 & 0.4 & 0.6 & 0.8 & 0.4 & 0.4 & 0.7 & 0.8 & 0.2 & 0.2 & 0.3 & 0.5 & 0.4 & 0.4 & 0.6 & 0.6 & 0.5 & 0.5 & 0.7 & 1.1 \\
\hline $\mathrm{NH}_{4}^{+}$ & 2.0 & 0.2 & 8.0 & 1.1 & 3.5 & 0.4 & 12.0 & 1.0 & 1.2 & 0.1 & 3.7 & 0.3 & 1.6 & 0.2 & 6.3 & 0.7 & 1.5 & 0.2 & 9.2 & 1.9 \\
\hline $\mathrm{K}^{+}$ & 0.4 & 0.2 & 0.9 & 0.3 & 0.6 & 0.3 & 1.2 & 0.6 & 0.1 & 0.0 & 0.4 & 0.1 & 0.3 & 0.1 & 0.7 & 0.1 & 0.6 & 0.3 & 1.3 & 0.5 \\
\hline $\mathrm{Mg}^{2+}$ & 0.2 & 0.3 & 0.2 & 0.5 & 0.2 & 0.4 & 0.2 & 0.5 & 0.2 & 0.3 & 0.1 & 0.4 & 0.2 & 0.4 & 0.2 & 0.5 & 0.2 & 0.3 & 0.2 & 0.4 \\
\hline $\mathrm{Ca}^{2+}$ & 1.0 & 3.1 & 1.0 & 3.9 & 1.0 & 3.8 & 1.0 & 5.4 & 0.6 & 2.5 & 0.7 & 3.1 & 1.0 & 3.3 & 1.1 & 4.2 & 1.1 & 2.2 & 1.1 & 2.9 \\
\hline
\end{tabular}




\begin{tabular}{|c|c|c|c|c|c|c|c|c|c|c|c|c|c|c|c|c|c|c|c|c|}
\hline $\mathrm{Cl}^{-}$ & 0.9 & 0.4 & 2.2 & 1.2 & 0.8 & 0.8 & 2.5 & 1.7 & 0.1 & 0.1 & 0.1 & 0.4 & 1.1 & 0.3 & 1.4 & 0.5 & 0.9 & 0.4 & 3.8 & 1.9 \\
\hline $\mathrm{NO}_{3}^{-}$ & 2.1 & 1.3 & 11.4 & 4.1 & 3.4 & 2.9 & 11.8 & 5.3 & 0.3 & 1.2 & 2.0 & 3.2 & 2.0 & 0.6 & 8.4 & 3.0 & 1.5 & 0.7 & 18.7 & 4.9 \\
\hline $\mathrm{SO}_{4}{ }^{2-}$ & 2.9 & 0.9 & 13.1 & 3.0 & 7.3 & 1.5 & 14.9 & 3.8 & 2.4 & 0.3 & 8.3 & 1.1 & 1.1 & 0.7 & 11.6 & 2.5 & 2.0 & 0.5 & 15.7 & 3.9 \\
\hline $\mathrm{Na}$ & 1.7 & 1.4 & 1.9 & 1.5 & 1.9 & 1.7 & 1.9 & 1.5 & 1.7 & 0.8 & 1.7 & 1.2 & 1.6 & 1.3 & 1.9 & 1.3 & 1.6 & 1.5 & 1.9 & 1.7 \\
\hline $\mathrm{Mg}$ & 0.5 & 1.1 & 0.4 & 1.4 & 0.7 & 2.2 & 0.5 & 2.0 & 0.5 & 0.8 & 0.5 & 1.4 & 0.5 & 0.8 & 0.4 & 1.3 & 0.3 & 0.7 & 0.3 & 1.0 \\
\hline $\mathrm{Al}$ & 0.7 & 1.5 & 0.6 & 1.5 & 0.9 & 3.3 & 0.7 & 2.4 & 0.7 & 0.8 & 0.5 & 1.0 & 0.7 & 1.2 & 0.7 & 1.4 & 0.5 & 1.0 & 0.6 & 1.1 \\
\hline $\mathrm{K}$ & 0.4 & 0.5 & 0.9 & 0.8 & 0.7 & 1.4 & 1.0 & 1.2 & 0.3 & 0.3 & 0.5 & 0.6 & 0.3 & 0.3 & 0.9 & 0.8 & 0.2 & 0.4 & 1.0 & 0.5 \\
\hline $\mathrm{Ca}$ & 3.5 & 10.7 & 3.4 & 10.6 & 5.6 & 29.5 & 3.5 & 17.7 & 4.9 & 7.4 & 5.1 & 13.0 & 2.8 & 6.8 & 3.6 & 9.3 & 2.3 & 5.5 & 2.3 & 5.9 \\
\hline $\mathrm{Mn}$ & 0.03 & 0.04 & 0.04 & 0.05 & 0.04 & 0.10 & 0.05 & 0.07 & 0.03 & 0.02 & 0.03 & 0.03 & 0.03 & 0.03 & 0.05 & 0.04 & 0.01 & 0.03 & 0.04 & 0.05 \\
\hline $\mathrm{Fe}$ & 1.13 & 2.48 & 1.09 & 2.93 & 2.15 & 6.48 & 1.34 & 4.07 & 1.81 & 1.66 & 1.04 & 2.24 & 0.92 & 1.65 & 1.45 & 3.08 & 0.35 & 1.45 & 0.61 & 2.36 \\
\hline Co & 0.001 & 0.001 & 0.001 & 0.001 & 0.001 & 0.002 & 0.001 & 0.002 & 0.000 & 0.001 & 0.000 & 0.001 & 0.000 & 0.001 & 0.001 & 0.001 & 0.000 & 0.001 & 0.001 & 0.001 \\
\hline $\mathrm{Ni}$ & 0.013 & 0.016 & 0.013 & 0.013 & 0.011 & 0.014 & 0.011 & 0.013 & 0.018 & 0.022 & 0.014 & 0.008 & 0.011 & 0.017 & 0.014 & 0.013 & 0.015 & 0.013 & 0.013 & 0.015 \\
\hline $\mathrm{Cu}$ & 0.019 & 0.020 & 0.034 & 0.022 & 0.029 & 0.030 & 0.034 & 0.022 & 0.012 & 0.013 & 0.016 & 0.014 & 0.018 & 0.021 & 0.037 & 0.024 & 0.015 & 0.015 & 0.038 & 0.024 \\
\hline $\mathrm{Zn}$ & 0.150 & 0.076 & 0.253 & 0.111 & 0.291 & 0.126 & 0.320 & 0.161 & 0.203 & 0.092 & 0.186 & 0.093 & 0.118 & 0.071 & 0.279 & 0.098 & 0.089 & 0.047 & 0.220 & 0.101 \\
\hline Mo & 0.001 & 0.001 & 0.002 & 0.002 & 0.002 & 0.001 & 0.001 & 0.001 & 0.001 & 0.002 & 0.002 & 0.001 & 0.001 & 0.001 & 0.002 & 0.002 & 0.002 & 0.001 & 0.003 & 0.002 \\
\hline $\mathrm{Cd}$ & 0.001 & 0.000 & 0.002 & 0.000 & 0.002 & 0.000 & 0.003 & 0.001 & 0.001 & 0.000 & 0.001 & 0.000 & 0.000 & 0.000 & 0.002 & 0.000 & 0.000 & 0.000 & 0.002 & 0.000 \\
\hline $\mathrm{Ba}$ & 0.016 & 0.039 & 0.017 & 0.050 & 0.022 & 0.080 & 0.016 & 0.066 & 0.012 & 0.026 & 0.013 & 0.035 & 0.015 & 0.032 & 0.019 & 0.052 & 0.014 & 0.029 & 0.018 & 0.045 \\
\hline $\mathrm{Tl}$ & 0.001 & 0.000 & 0.001 & 0.000 & 0.001 & 0.000 & 0.002 & 0.000 & 0.000 & 0.000 & 0.001 & 0.000 & 0.000 & 0.000 & 0.002 & 0.000 & 0.000 & 0.000 & 0.001 & 0.000 \\
\hline $\mathrm{Pb}$ & 0.047 & 0.012 & 0.134 & 0.029 & 0.114 & 0.032 & 0.209 & 0.056 & 0.029 & 0.006 & 0.083 & 0.014 & 0.033 & 0.009 & 0.105 & 0.017 & 0.025 & 0.006 & 0.136 & 0.030 \\
\hline Th & 0.000 & 0.000 & 0.000 & 0.001 & 0.000 & 0.001 & 0.000 & 0.001 & 0.000 & 0.000 & 0.000 & 0.000 & 0.000 & 0.000 & 0.000 & 0.000 & 0.000 & 0.000 & 0.000 & 0.001 \\
\hline $\mathrm{U}$ & 0.000 & 0.000 & 0.000 & 0.000 & 0.000 & 0.000 & 0.000 & 0.000 & 0.000 & 0.000 & 0.000 & 0.000 & 0.000 & 0.000 & 0.000 & 0.000 & 0.000 & 0.000 & 0.000 & 0.000 \\
\hline
\end{tabular}


Table S3 The correlation coefficients between chemical species in different size fractions and visibility

\begin{tabular}{ccccccccccc}
\hline Size & $\mathrm{OC}$ & $\mathrm{EC}$ & $\mathrm{Na}^{+}$ & $\mathrm{MH}_{4}{ }^{+}$ & $\mathrm{K}^{+}$ & $\mathrm{Mg}^{2+}$ & $\mathrm{Ca}^{2+}$ & $\mathrm{Cl}^{-}$ & $\mathrm{NO}_{3}{ }^{-}$ & $\mathrm{SO}_{4}{ }^{2-}$ \\
\hline$<0.43$ & -0.316 & -0.192 & -0.158 & $\mathbf{- 0 . 3 9 3}$ & -0.321 & -0.174 & -0.199 & -0.355 & $\mathbf{- 0 . 3 5 0}$ & $\mathbf{- 0 . 3 0 3}$ \\
$0.43-0.65$ & $\mathbf{- 0 . 3 7 1}$ & -0.189 & -0.113 & $\mathbf{- 0 . 5 0 6}$ & $\mathbf{- 0 . 3 8 0}$ & -0.215 & -0.248 & $\mathbf{- 0 . 4 1 4}$ & $\mathbf{- 0 . 4 4 5}$ & $\mathbf{- 0 . 5 9 0}$ \\
$0.65-1.1$ & $\mathbf{- 0 . 4 8 5}$ & -0.119 & -0.200 & $\mathbf{- 0 . 4 1 8}$ & $\mathbf{- 0 . 3 8 4}$ & -0.218 & -0.282 & $\mathbf{- 0 . 3 7 7}$ & $\mathbf{- 0 . 4 7 0}$ & $\mathbf{- 0 . 5 8 2}$ \\
$1.1-2.1$ & -0.296 & -0.173 & -0.180 & $\mathbf{- 0 . 4 1 4}$ & -0.356 & -0.199 & -0.279 & -0.308 & $\mathbf{- 0 . 3 5 9}$ & $\mathbf{- 0 . 3 8 8}$ \\
$2.1-3.3$ & -0.234 & -0.196 & -0.196 & -0.217 & -0.295 & -0.238 & -0.358 & $\mathbf{- 0 . 3 1 9}$ & $\mathbf{- 0 . 3 4 0}$ & $\mathbf{- 0 . 3 0 2}$ \\
$3.3-4.7$ & -0.207 & -0.209 & -0.139 & -0.218 & -0.255 & -0.196 & -0.421 & -0.195 & $\mathbf{- 0 . 4 3 3}$ & -0.255 \\
$4.7-5.8$ & -0.196 & -0.183 & -0.206 & -0.242 & -0.189 & -0.245 & -0.481 & -0.258 & $\mathbf{- 0 . 4 0 6}$ & -0.232 \\
$5.8-9$ & -0.354 & -0.123 & -0.218 & -0.318 & -0.193 & $\mathbf{- 0 . 4 2 6}$ & $\mathbf{- 0 . 5 2 0}$ & $\mathbf{- 0 . 3 1 1}$ & $\mathbf{- 0 . 5 0 7}$ & $\mathbf{- 0 . 4 6 1}$ \\
$>9$ & -0.477 & -0.145 & -0.185 & -0.245 & -0.183 & -0.319 & -0.388 & -0.195 & $\mathbf{- 0 . 4 2 5}$ & $\mathbf{- 0 . 3 4 6}$ \\
\hline
\end{tabular}

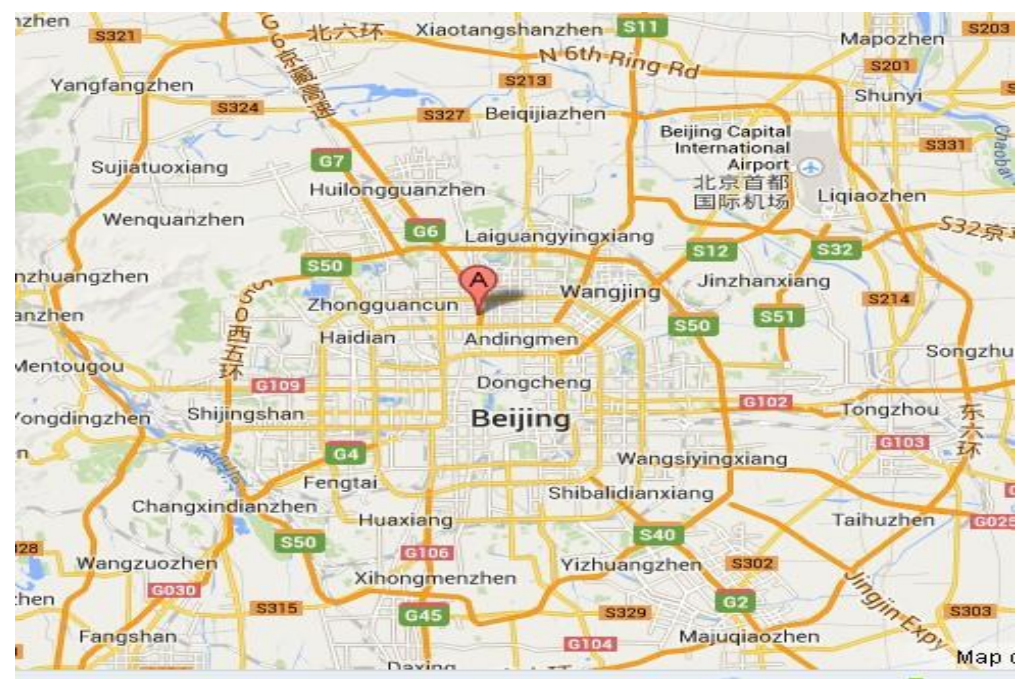

Fig. S1 Map of the site location in Beijing (A was the sampling site, http://www.google.cn/maps). 

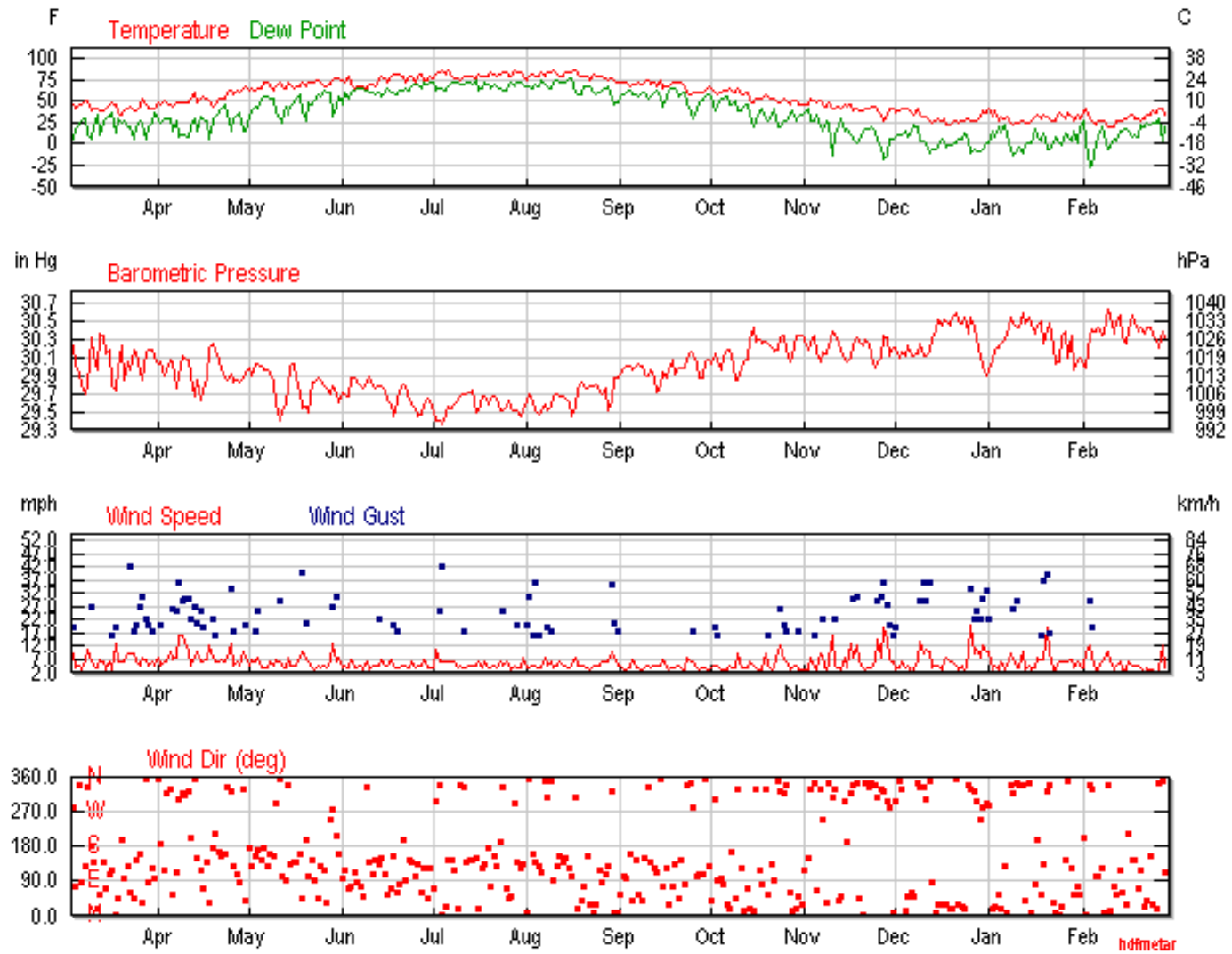

Fig. S2 Meteorological parameters in Beijing from March 1, 2013, to February 28,

2014 (http://english.wunderground.com)
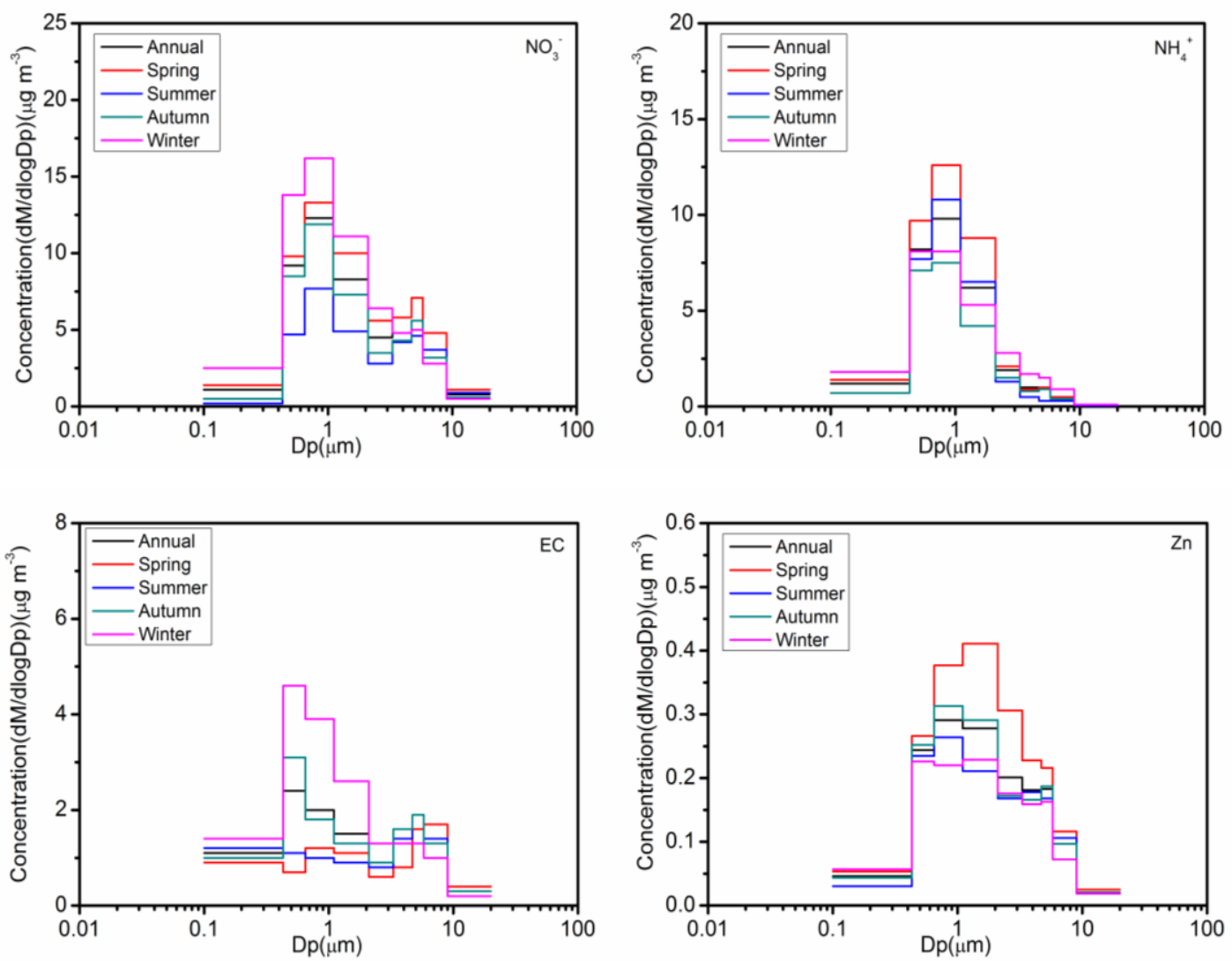

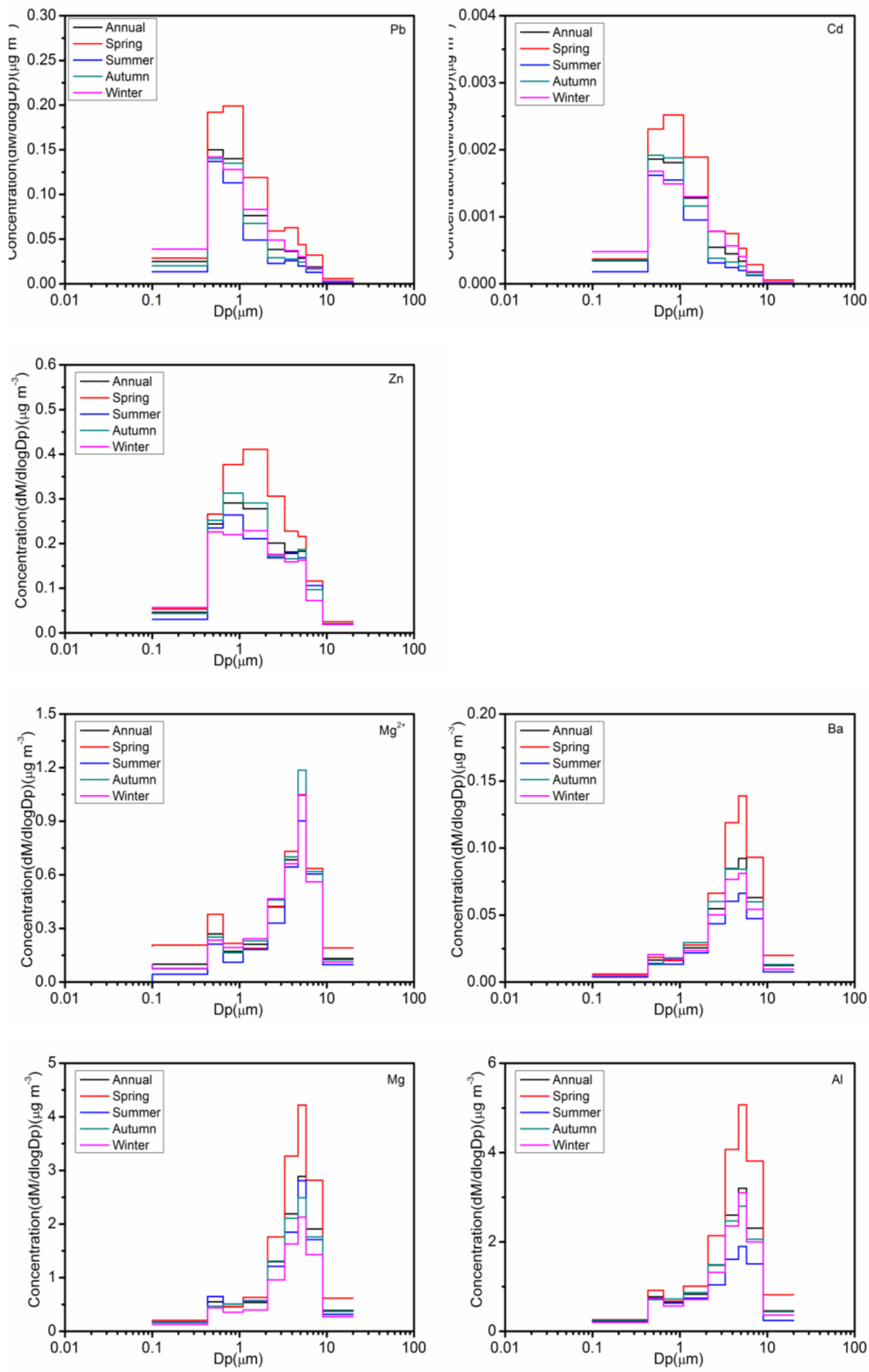

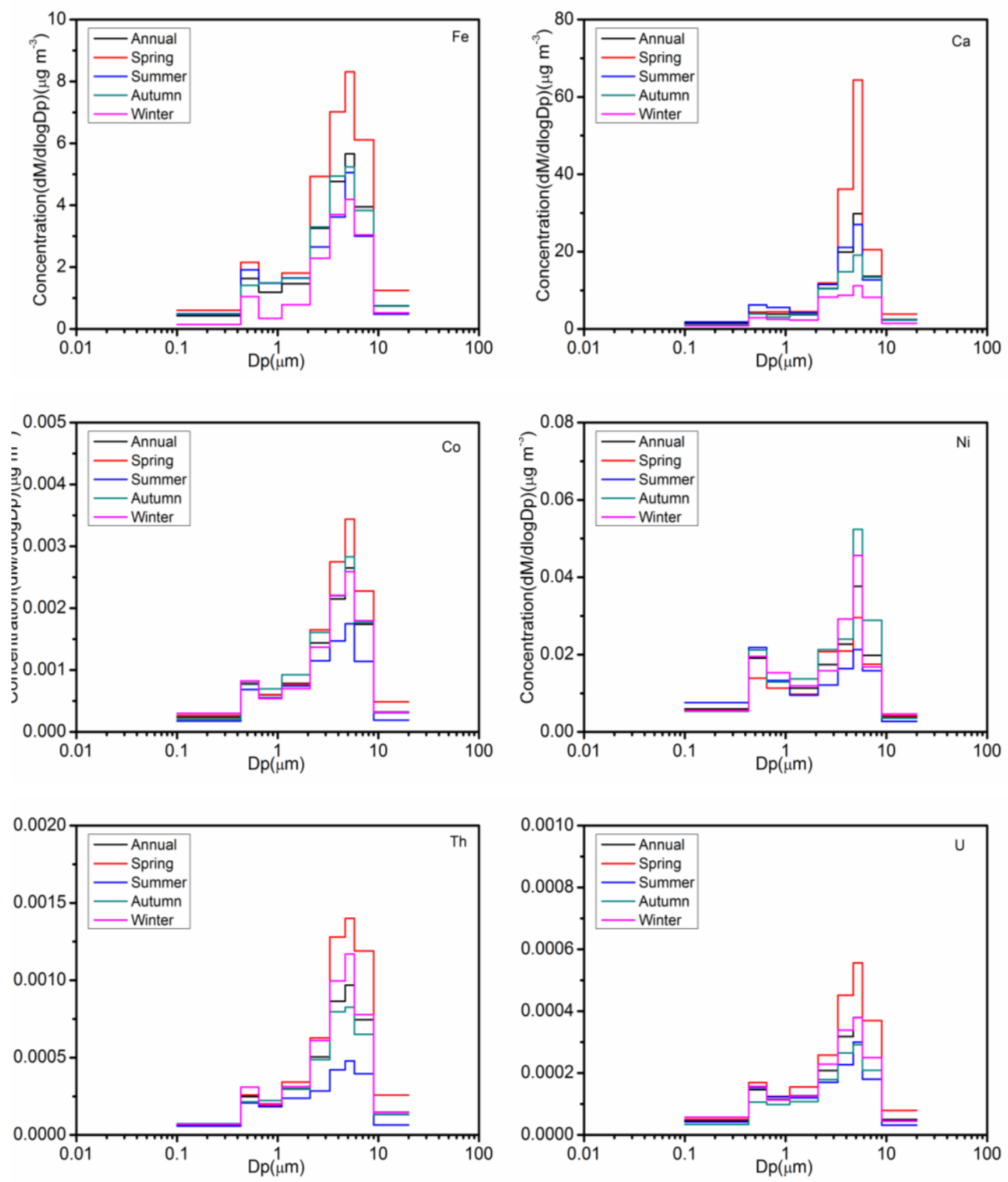

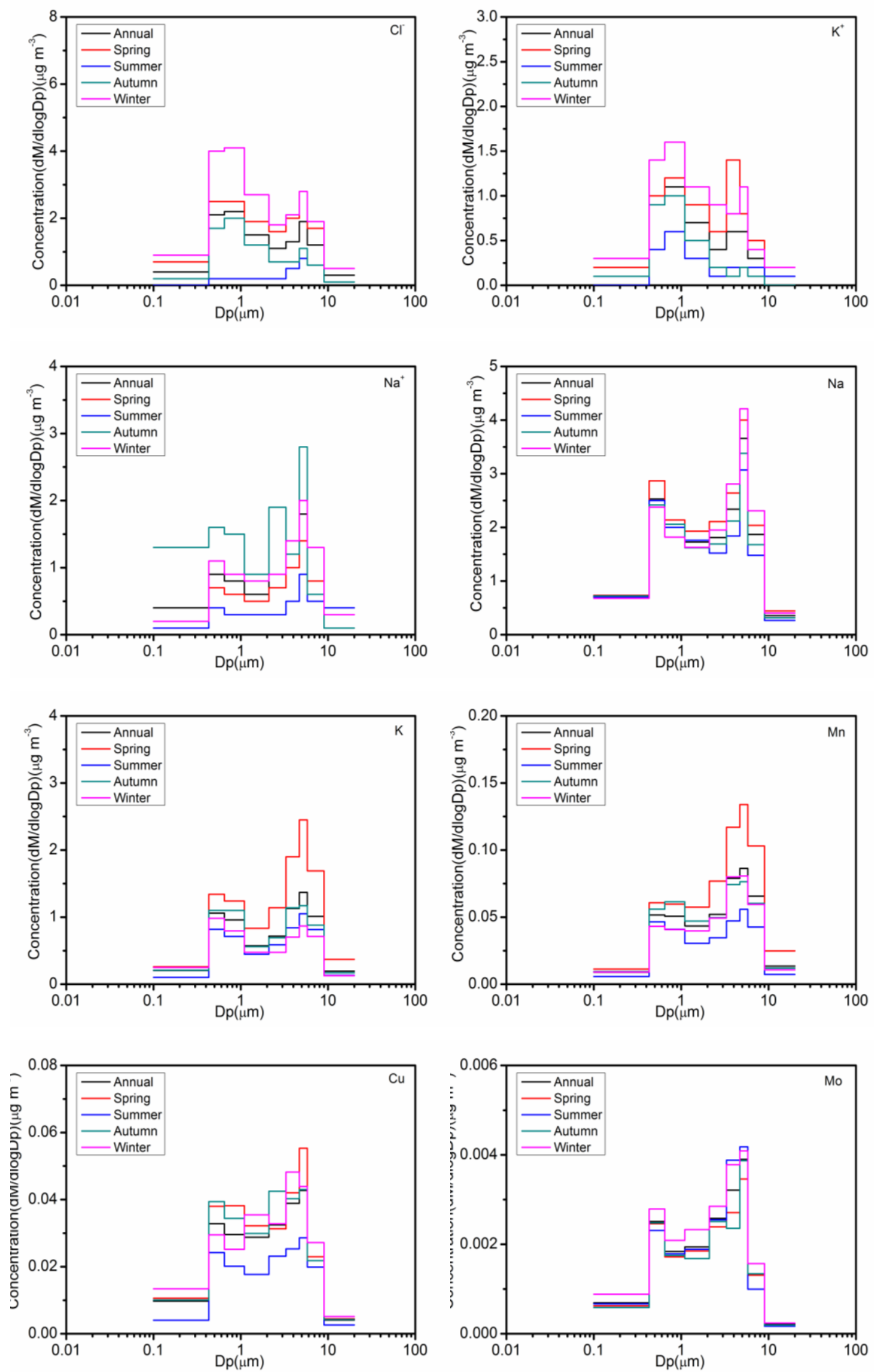

Fig. S3 Size distributions of chemical species in different seasons. 


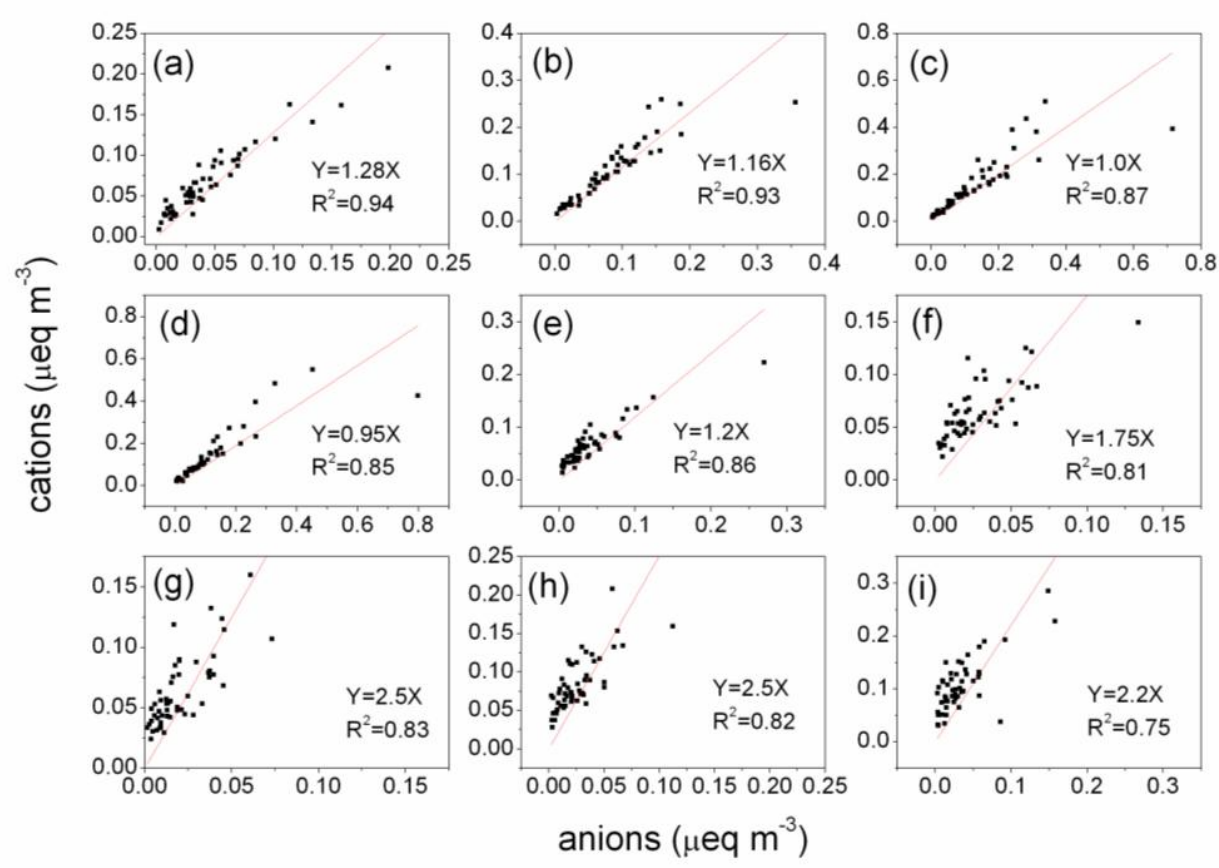

Fig. S4 Ion balance for different size fractions: (a) $<0.43 \mu \mathrm{m}$; (b) $0.43-0.65 \mu \mathrm{m}$; (c)

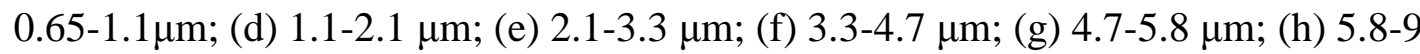
$\mu \mathrm{m}$; (i) $>9 \mu \mathrm{m}$.
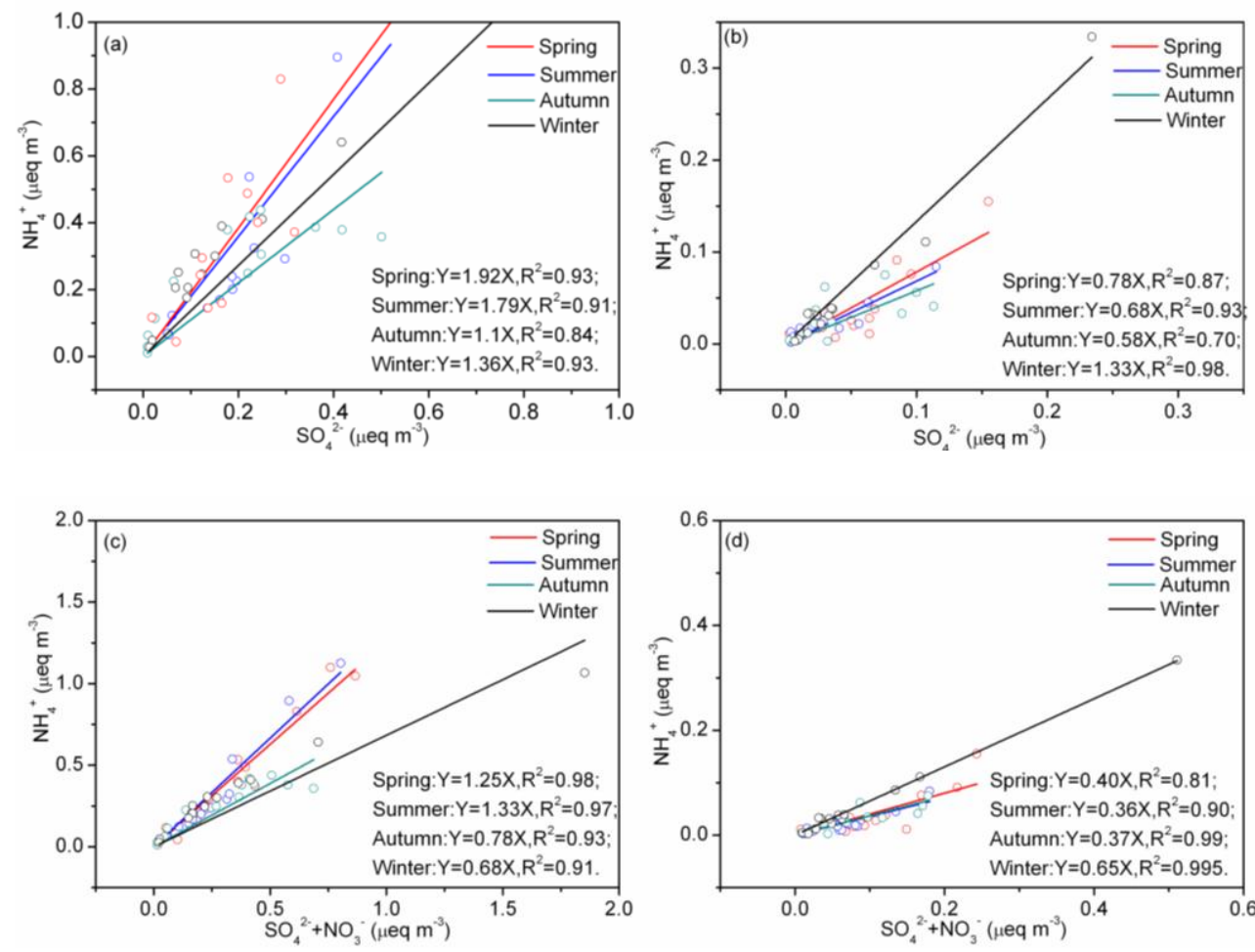

Fig. S5 Correlations between certain cations and anions in both fine and coarse particles during different seasons: (a) $\mathrm{NH}_{4}{ }^{+}$and $\mathrm{SO}_{4}{ }^{2-}$ in fine particles; (b) $\mathrm{NH}_{4}{ }^{+}$and $\mathrm{SO}_{4}{ }^{2-}$ in coarse particles; (c) $\mathrm{NH}_{4}{ }^{+}$to $\left[\mathrm{NO}_{3}{ }^{-}+\mathrm{SO}_{4}{ }^{2-}\right]$ in fine particles; ; (d) $\mathrm{NH}_{4}{ }^{+}$to $\left[\mathrm{NO}_{3}{ }^{-}+\mathrm{SO}_{4}{ }^{2-}\right]$ in coarse particles. 

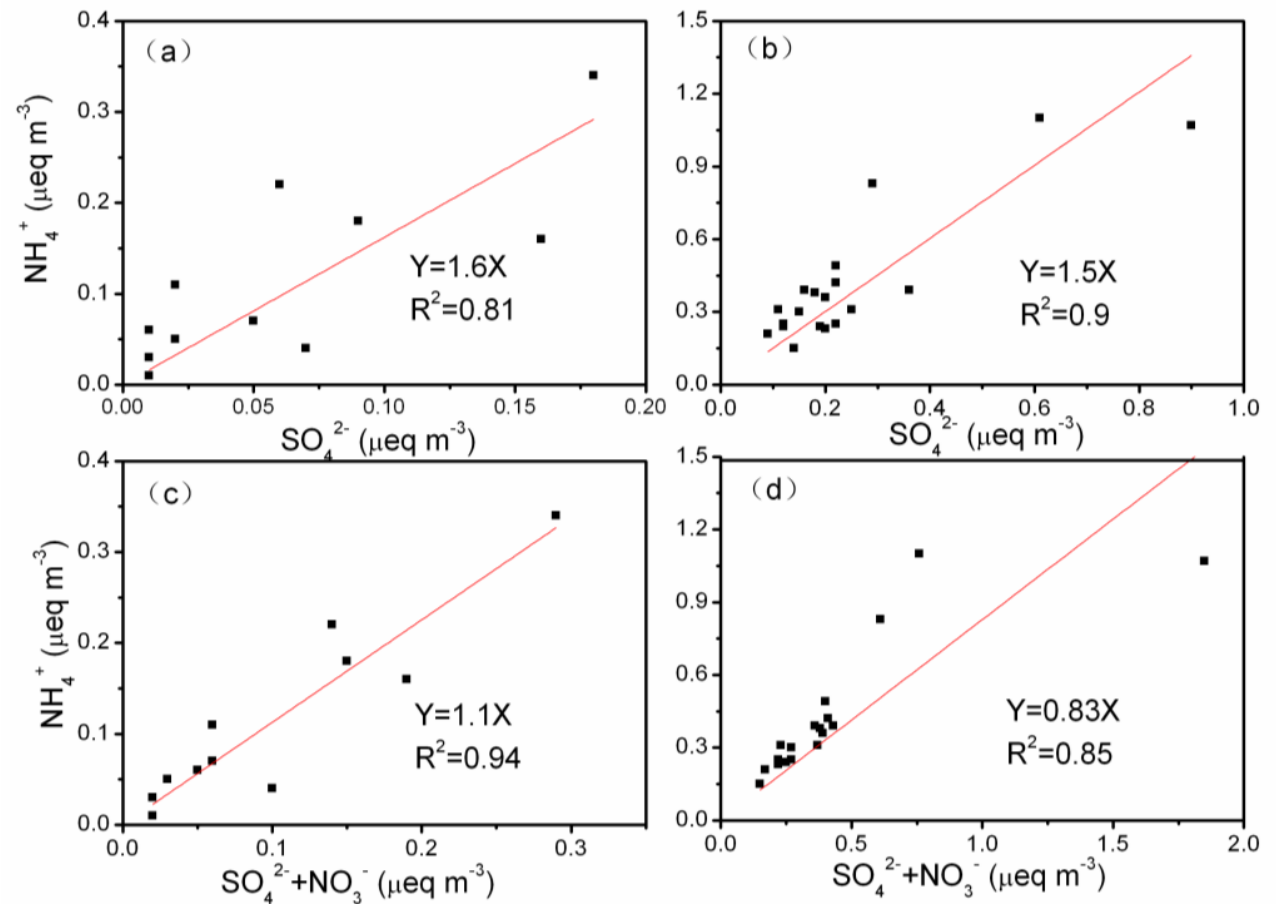

Fig. S6 Correlations between certain cations and anions in fine particles on both nonhaze and haze days: (a) $\mathrm{NH}_{4}{ }^{+}$and $\mathrm{SO}_{4}{ }^{2-}$ on non-haze days; (b) $\mathrm{NH}_{4}{ }^{+}$and $\mathrm{SO}_{4}{ }^{2-}$ on haze days; (c) $\mathrm{NH}_{4}{ }^{+}$to $\left[\mathrm{NO}_{3}{ }^{-}+\mathrm{SO}_{4}{ }^{2-}\right]$ on non-haze days; (d) $\mathrm{NH}_{4}{ }^{+}$to $\left[\mathrm{NO}_{3}{ }^{-}+\mathrm{SO}_{4}{ }^{2-}\right]$ on haze days.
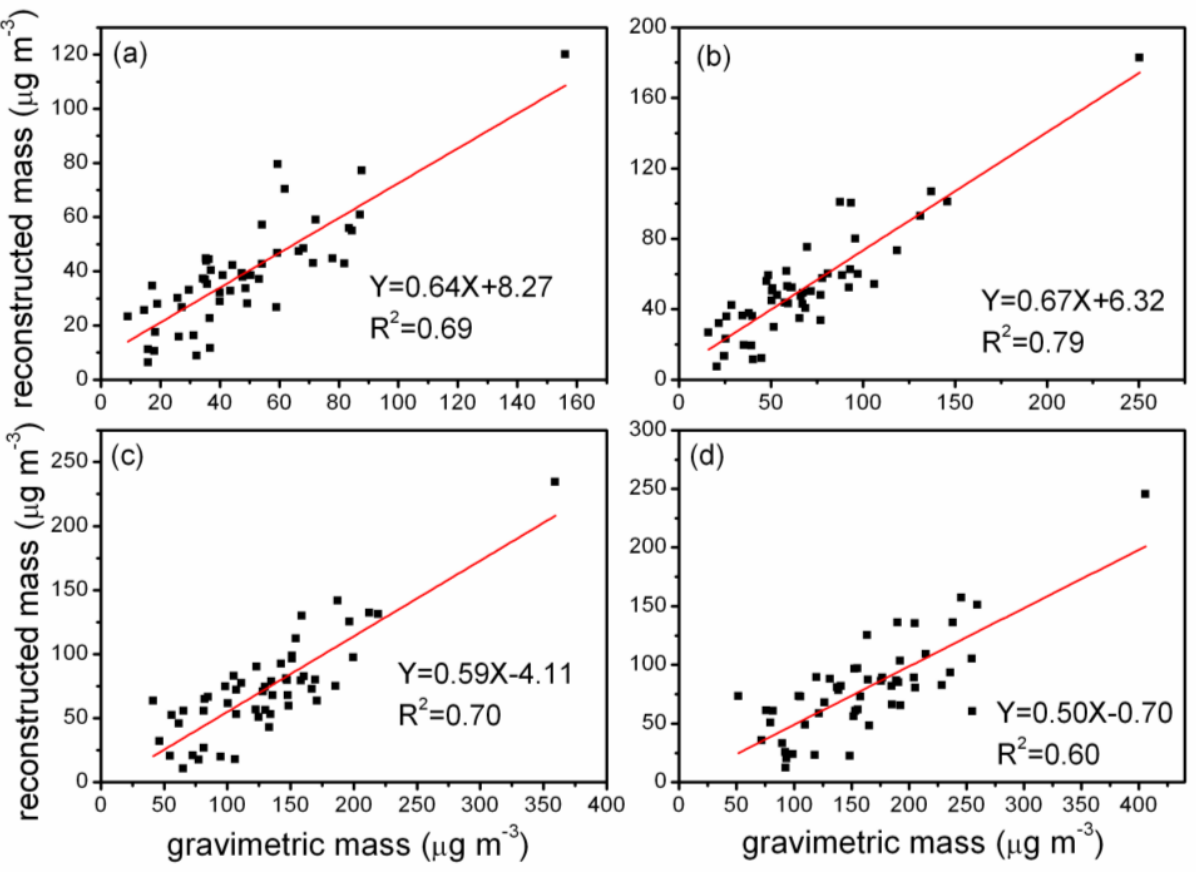

Fig.S7 The relationship between reconstructed PM mass concentrations and the 
gravimetric PM mass concentrations: (a) $\mathrm{PM}_{1.1}$; (b) $\mathrm{PM}_{2.1}$; (c) $\mathrm{PM}_{9}$; (d) TSP.

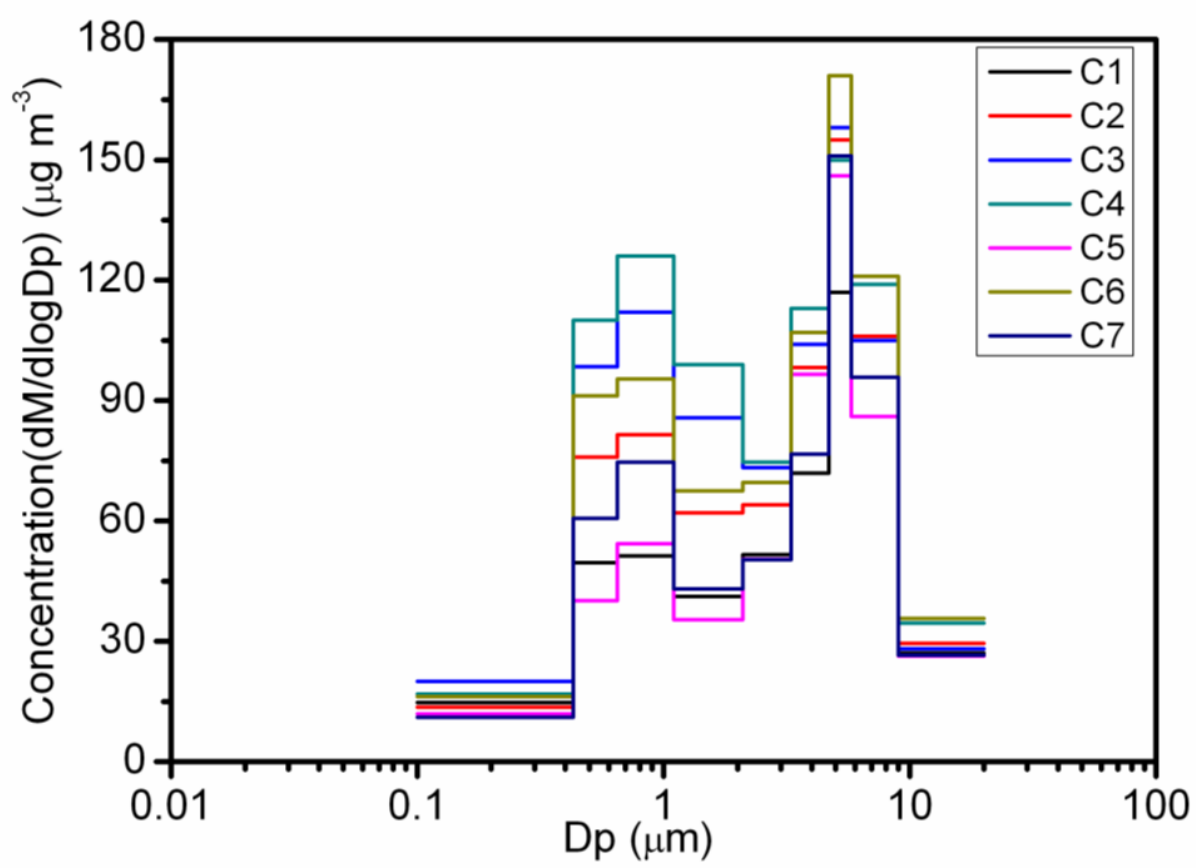

Fig. S8 Mass concentration size distributions within each trajectory cluster.
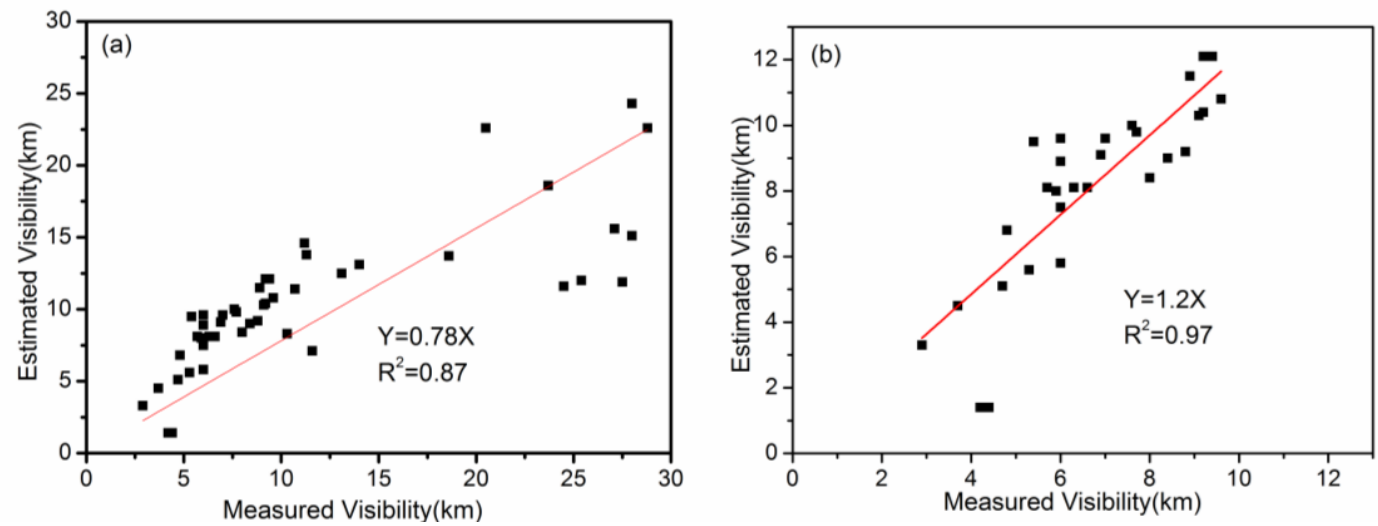

Fig. S9 Correction between estimated visibility and measured visibility (a) all the datasets in 2012-2013; (b) datasets with visibility lower than $10 \mathrm{~km}$ in 2012-2013.

\section{References}

Clegg, S. L., Brimblecombe, P., and Wexler, A. S.: Thermodynamic model of the system $\mathrm{H}^{+}-\mathrm{NH}_{4}{ }^{+}-\mathrm{SO}_{4}{ }^{2-}-\mathrm{NO}_{3}{ }^{-}-\mathrm{H}_{2} \mathrm{O}$ at tropospheric temperatures, J Phys Chem A, 102 , 2137-2154, 1998. 
Hueglin, C., Gehrig, R., Baltensperger, U., Gysel, M., Monn, C., and Vonmont, H.: Chemical characterisation of $\mathrm{PM}_{2.5}, \mathrm{PM}_{10}$ and coarse particles at urban, near-city and rural sites in Switzerland, Atmos Environ, 39, 637-651, 2005.

Terzi, E., Argyropoulos, G., Bougatioti, A., Mihalopoulos, N., Nikolaou, K., and Samara, C.: Chemical composition and mass closure of ambient $\mathrm{PM}_{10}$ at urban sites, Atmos Environ, 44, 2231-2239, 2010. 